\title{
Editorial
}

\section{Metal Oxide Nanostructures: Synthesis, Properties, and Applications}

\author{
Lin-Hua Xu, ${ }^{1}$ Dnyaneshwar S. Patil, ${ }^{2}$ Jiazhi Yang, ${ }^{3}$ and Jingzhong Xiao ${ }^{4}$ \\ ${ }^{1}$ School of Physics and Optoelectronic Engineering, Nanjing University of Information Science \& Technology, Nanjing 210044, China \\ ${ }^{2}$ Department of Electronics, North Maharashtra University, Jalgaon, Maharashtra 425001, India \\ ${ }^{3}$ School of Chemistry and Chemical Engineering, Nanjing University of Science and Technology, Nanjing 210094, China \\ ${ }^{4}$ Department of Physics, Faculty of Science and Technology (FCT), University of Coimbra (UC), Rua Larga, \\ 3004-516 Coimbra, Portugal
}

Correspondence should be addressed to Lin-Hua Xu; 474860864@qq.com

Received 7 September 2015; Accepted 7 September 2015

Copyright (C) 2015 Lin-Hua Xu et al. This is an open access article distributed under the Creative Commons Attribution License, which permits unrestricted use, distribution, and reproduction in any medium, provided the original work is properly cited.

In recent years, nanostructured materials have attracted wide attention due to their fascinating optical and electrical properties, which make these materials potentially suitable for applications in electronics, optics, photonics, and sensors. Some metal oxides show a wide variety of morphologies such as nanowires, nanorods, nanotubes, nanorings, and nanobelts. Synthesis and investigation of these metal-oxide nanostructures are beneficial not only for understanding the fundamental phenomena in low dimensional systems, but also for developing new-generation nanodevices with high performance.

This special issue is focused on synthesis, properties, characterizations, and numerical modeling of metal-oxide nanomaterials and nanostructures with potential applications in photocatalysts, wastewater purification, chemical sensors, and so on.

B. Dai et al. reported that an environment-friendly biomaterial bacterial cellulose (BC) was introduced to substitute for general organic polymers to assist the preparation of $\mathrm{ZnO} / \mathrm{CdS} / \mathrm{BC}$ photocatalyst hybrid nanofiber through coprecipitation method under the low-temperature condition. The photocatalytic activities of $\mathrm{ZnO} / \mathrm{CdS} / \mathrm{BC}$ were influenced by the added amount of $\mathrm{CdS}$. It is found that $\mathrm{ZnO} / \mathrm{CdS} / \mathrm{BC}$ nanohybrid fibers (CdS: 10\%) show the best photocatalytic efficiency among the samples.

R. Liu and H. Tai Ou reported that IC lead frame scraps were used in their research as raw materials to fabricate magnetic ferrite powders and combined subsequently with titanium sulfate and urea to produce magnetic photocatalysts by coprecipitation for effective waste utilization. They found that wastes can be transformed to valuable magnetic photocatalysts in their research to solve the separation problem of wastewater and $\mathrm{TiO}_{2}$ photocatalysts by magnetic field.

M. Mahlambi et al. studied the $\mathrm{TiO}_{2}$ nanocatalysts supported on a hybrid carbon-covered alumina (CCA) support and carried out a comparison between visible light and UV light degradation of Rhodamine $\mathrm{B}$. The $\mathrm{CCA} / \mathrm{TiO}_{2}$ nanocatalysts had a much larger surface area than the unsupported titania and they exhibited overall higher photodegradation efficiency under both UV and visible light than unsupported $\mathrm{TiO}_{2}$.

T. Le et al. reported that the enzyme CPO was successfully entrapped inside a silica nanostructure prepared from the precursor TMOS with or without addition of the hydrophobic modifier MTMS. The catalytic performance of optimized CPO sol-gel beads approached $18 \%$ relative to free CPO in solution as assessed via the pyrogallol peroxidation assay.

M. Daud et al. carried out a research work for the removal of nitrate from raw water for a drinking water supply using $\mathrm{Fe} / \mathrm{Pt}$ bimetallic nanoparticles and anion exchange resins. The overall results indicate that $\mathrm{Fe} / \mathrm{Pt}$ nanoparticles having a large surface area $\left(627 \mathrm{~m}^{2} / \mathrm{g}\right)$ are potentially more efficient than the commercially used anionic resins for nitrate removal from water. 
Growth mechanisms of nanostructured titania in turbulent reacting flows were studied by S. C. Garrick. Direct numerical simulation is utilized in simulating the hydrolysis of titanium tetrachloride to produce titania in a turbulent, planar jet. Results show that, in the proximal region of the jet, nucleation and condensation are the dominant mechanisms. However, once the jet potential core collapses and turbulent mixing begins, coagulation is the dominant mechanism. The data also shows that the coagulation growth rate is as much as twice the condensation growth rate.

\section{Acknowledgments}

The guest editorial team would like to thank the authors for their contributions to this special issue and the reviewers for their precious time and dedication.

Lin-Hua Xu

Dnyaneshwar S. Patil

Jiazhi Yang

Jingzhong Xiao 

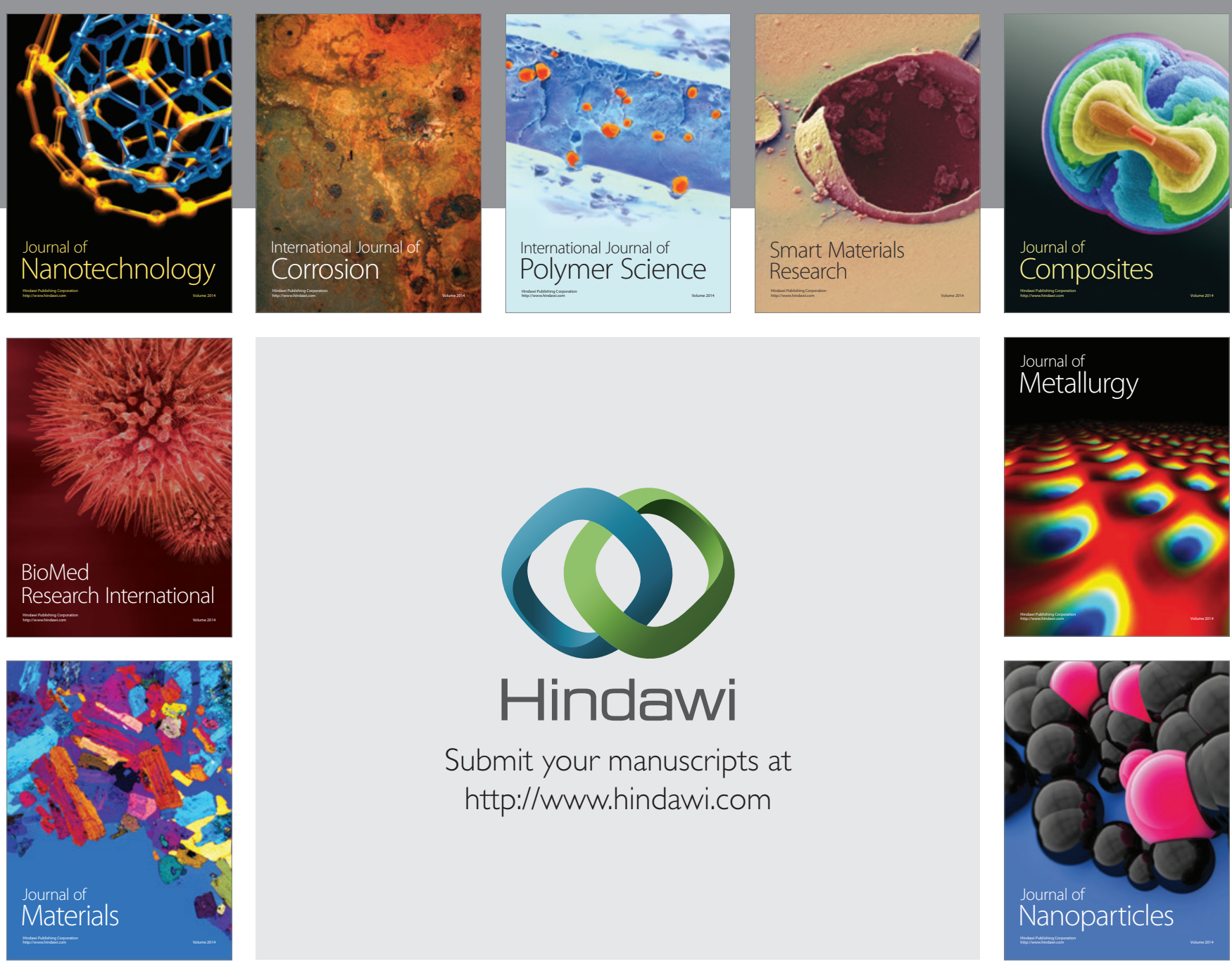

Submit your manuscripts at http://www.hindawi.com
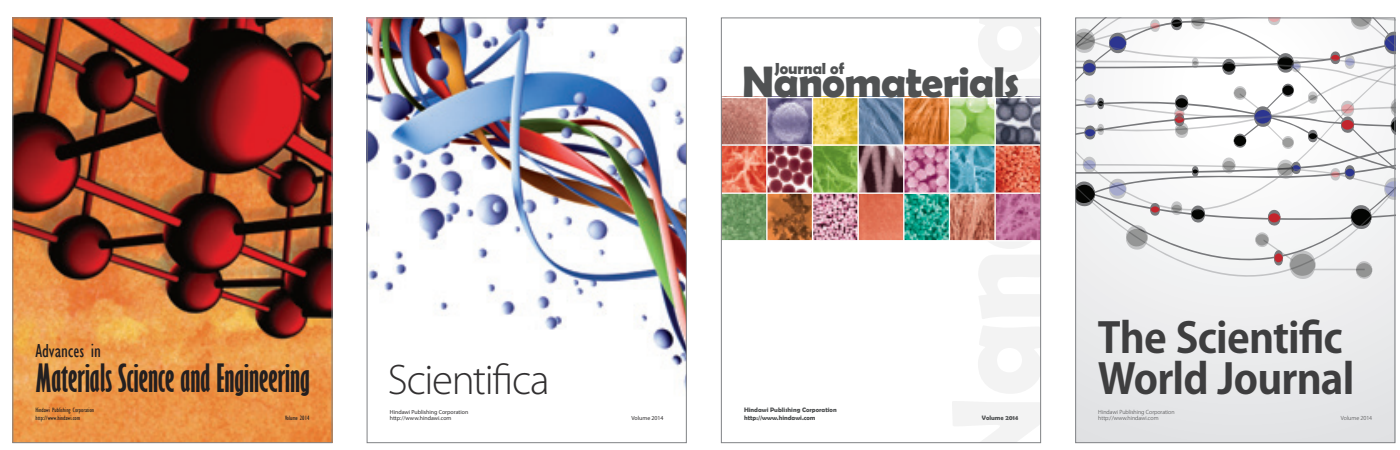

\section{The Scientific World Journal}
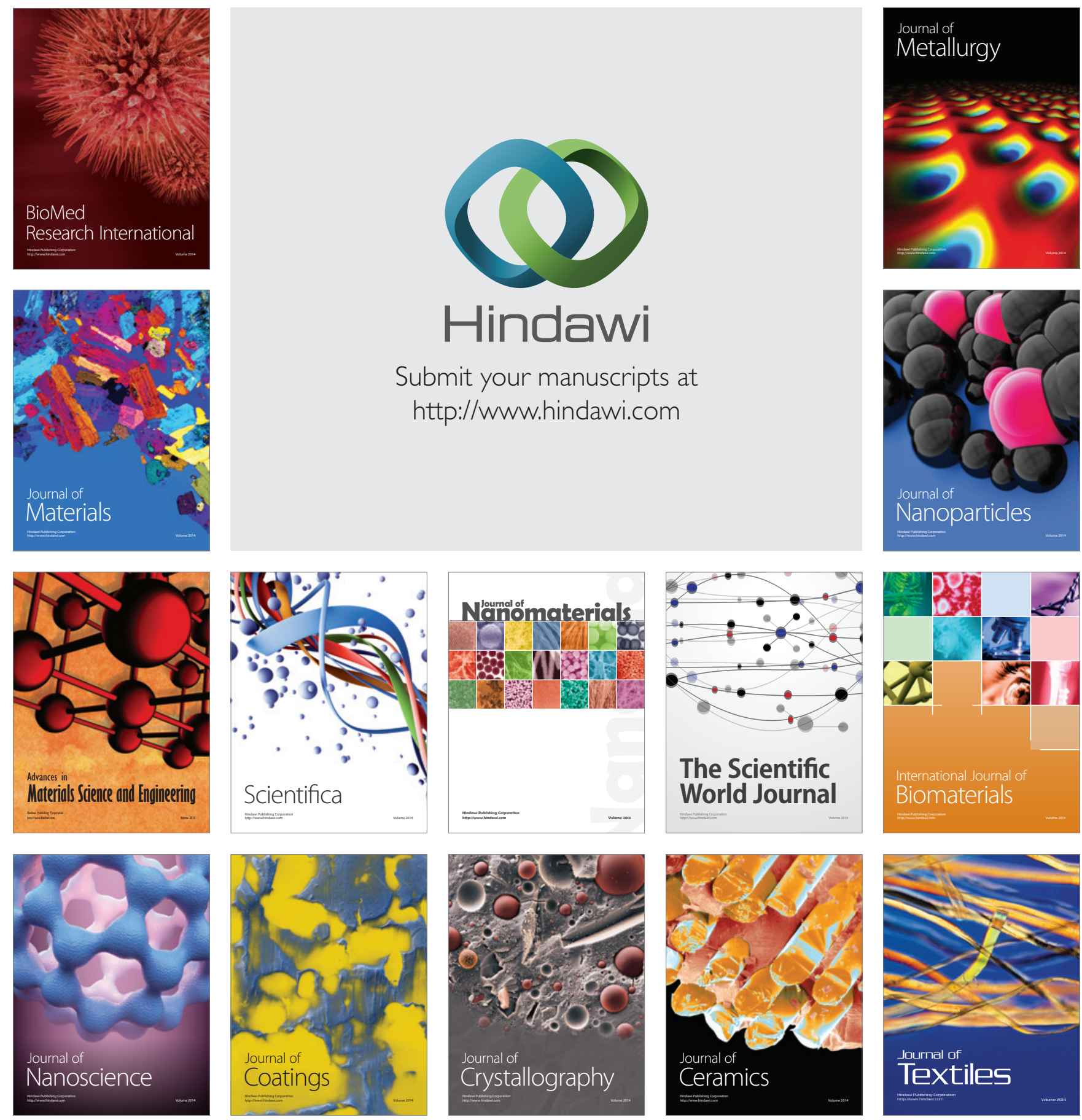